\section{Brain, Behavior and Evolution}

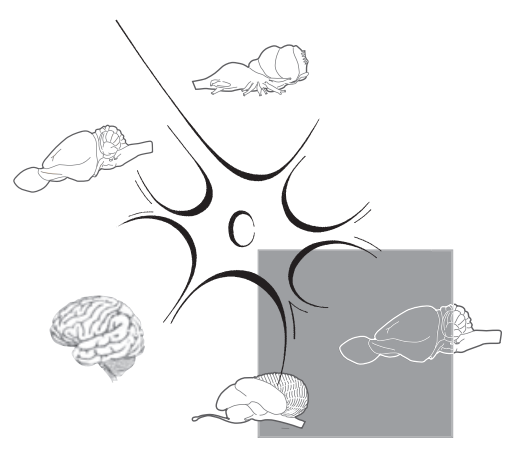

\section{Introduction}

The cerebral hemispheres are the most expanded brain region in most vertebrate lineages, and these expansions are generally associated with increases in behavioral complexity. In association with these expansions, the dorsal component of the hemispheres (the pallium) develops diverging morphologies in the various vertebrate classes, making it very difficult to establish correspondences between groups. The most likely interpretation is that brain expansion occurred separately in the different vertebrate classes, taking advantage of different embryological mechanisms [Striedter, 2005; Aboitiz and Montiel, 2012]. The best-studied vertebrates in this sense are birds and mammals, which have both developed large brains and elaborate cognitive abilities. Mammals are characterized by a conspicuous 6-layered isocortex, and reptiles and birds display a large, globular structure that bulges into the lateral ventricle, called the dorsal ventricular ridge (DVR). In birds, this structure hypertrophies and is subdivided into several components, called mesopallium, nidopallium, and arcopallium, plus some subdivisions within them (Fig. 1a). Above the DVR in

\title{
Homology in Amniote Brain Evolution: The Rise of Molecular Evidence
}

\author{
Juan F. Montiel ${ }^{a, b}$ Francisco Aboitiz ${ }^{c}$ \\ a Instituto de Ciencias de la Salud, Universidad de O'Higgins, Rancagua, Chile; \\ ${ }^{b}$ Universidad Diego Portales, Santiago, Chile; ' ${ }^{C}$ Centro Interdisciplinario de Neurociencias, \\ Escuela de Medicina, Pontificia Universidad Católica de Chile, Santiago, Chile
}

reptiles, there are a small dorsal cortex and a hippocampus, the former growing into a structure called hyperpallium in birds [Striedter, 1997]. Comparing these two types of pallial organizations and establishing homologies between them has been a major challenge for evolutionary neuroanatomy for about a century. Recently, high-throughput analyses of all active transcripts have become a powerful method for comparing brain regions among species and for inferring homologies. Novel RNA sequence technologies and analytic capacities enable us to investigate regional identities through unbiased comparisons across species using the whole transcriptome [Belgard et al., 2013; Belgard and Montiel, 2013; Pfenning et al., 2014; Montiel et al., 2016].

\section{The Search for Homology}

Early neuroanatomists analyzed the embryonic pallium of reptiles and mammals, and considered the DVR of birds and reptiles (sauropsids) to correspond to the ventral aspect of the pallium [Holmgren, 1922]. This idea was temporarily discarded when the DVR became associated to the corpus striatum in the subpallium, by virtue of the histological similarity between the two structures [Ariëns-Kappers et al., 1936]. A radical reinterpretation came with the groundbreaking hodological studies of Harvey Karten [1968, 1969], who showed that regions of the avian DVR (particularly, the nidopallium) received auditory and some visual projections comparable to those of the mammalian auditory and extrastriate visual cortices. This discovery firmly established the DVR as a pallial structure. In addition, the targets of primary visual and somatosensory projections in the dorsal pallium (dorsal cortex/hyperpallium) of sauropsids were considered comparable to the mammalian primary visual and somatosensory cortices. Thus, Karten proposed that mammalian isocortex had a dual origin, one being the DVR and the other the dorsal pallium. Given that the mammalian isocortex and the sauropsid DVR occupy

Our article is a commentary on the study by Briscoe SD, Albertin CB, Rowell JJ, Ragsdale CW (2018): Neocortical association cell types in the forebrain of birds and alligators. Curr Biol 28:686-696.e6.

\section{KARGER}

(c) 2018 S. Karger AG, Basel
Francisco Aboitiz

Departamento de Psiquiatría, Escuela de Medicina

Pontificia Universidad Católica de Chile

Marcoleta 391, Santiago (Chile)

E-Mail faboitiz@puc.cl 
Fig. 1. Pallial expression profiles and protein-protein interactions of mesopallial transcription factors. a Chicken and mouse gene expression comparisons for ID2, BCL11A, FOXP1, SATB2, and EMX1 transcription factors as determined from the website http://genserv.anat.ox.ac.uk/brainevo, provided by Belgard et al. [2013]. These genes exhibit a variable expression pattern across telencephalic compartments outside of the mesopallium. A, arcopallium; Ag, pallial amygdala; $\mathrm{Cl} / \mathrm{E}$, claustrum/ endopiriform complex; dCX, dorsal cortex; ICX, lateral cortex; DVR, dorsal ventricular ridge; $\mathrm{H}$, hyperpallium; $\mathrm{Hp}$, hippocampus; M, mesopallium; S, striatum; A-F are laminar cortical subdivisions of dCX and ICX (please refer to the website for laminar equivalents). Dashed line in the chicken brain represents the former $\mathrm{H} / \mathrm{M}$ division at the lamina mesopallium intermediate. However, gene expression largely supports a new border (solid line) involving the hyperpallium densocellulare in the dorsal mesopallium [Jarvis et al., 2013; Montiel and Molnár, 2013; Briscoe et al., 2018]. b Mesopallial transcription factors were analyzed using STRING 10.5 (http:// string-db.org/), a database of known and predicted protein associations, which responds by displaying a network of nodes (genes) connected by edges representing functional relationships, but not necessarily physical interactions. These transcriptional factors do not initially interact directly among them. SATB2/BCL11A/ NHLH2, and ID2/FOXP1 represent independent functional networks, joining only when one allows for multiple interactors/ proteins. Adding 20 direct interactors to the network, EMX1 remains as an unconnected node, and it remains so after adding an extra set/shell of 20 indirect interactions (data not shown).
CHICKEN BRAIN

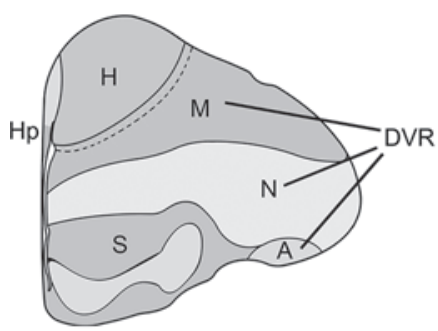

ID2

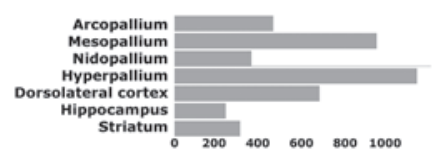

SATB2

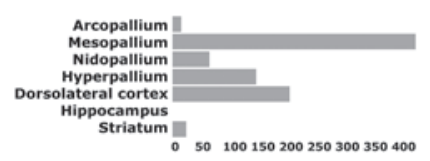

FOXP1

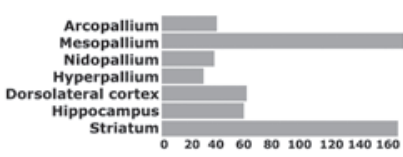

BCL11A

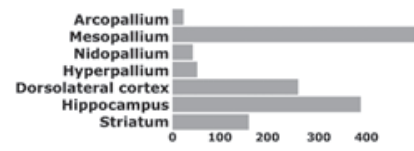

EMX1

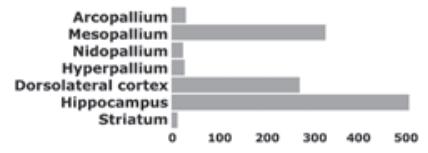

a
MOUSE BRAIN
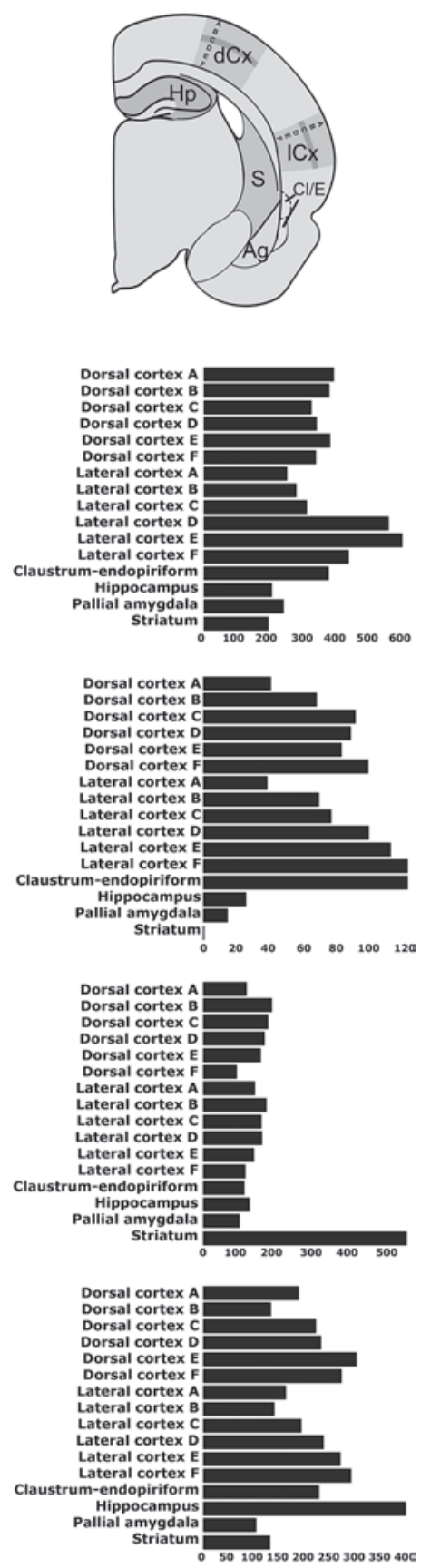

Dorsal cortex A Dorsal cortex B Dorsal cortex $\mathrm{C}$ Dorsal cortex $\mathrm{E}$ Dorsal cortex F Lateral cortex B Lateral cortex $\mathrm{D}$ Lateral cortex E
Lateral cortex $\mathrm{F}$ Claustrum-endopiriform
Hippocampus Hippocampus
Pallial amygdala Striatum

$$
\text { อ } 20406080100120140160
$$

(Figure continued on next page.) 


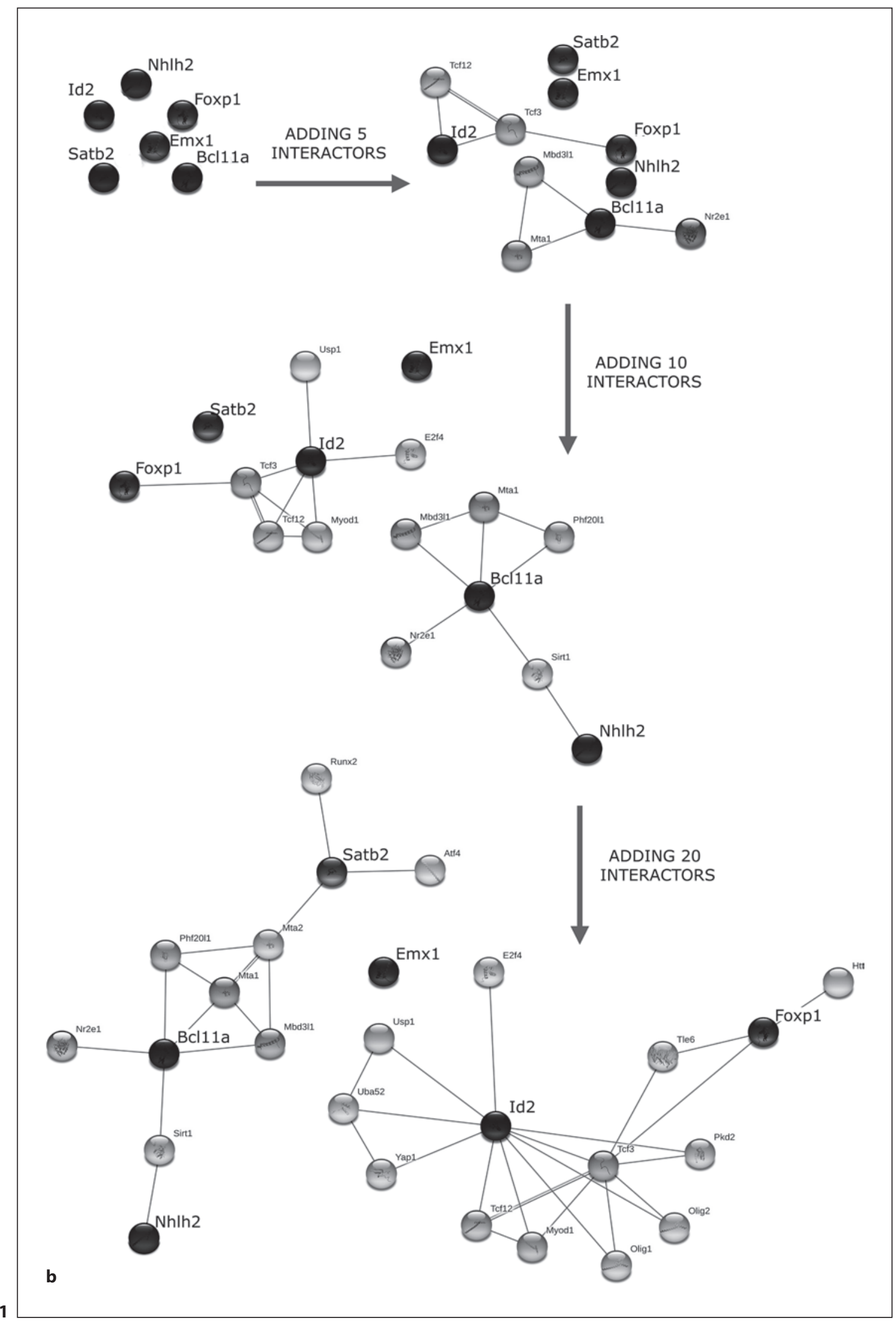


different positions within the cerebral hemispheres, Karten hypothesized that a major neuronal migration of excitatory cells from the ventral aspect of the pallium to more dorsal regions gave rise to the auditory and extrastriate visual cortices in mammals [Karten, 1997]. In addition, he proposed that the basic neural processing circuit, from input-receiving neurons to output neurons, with interneurons connecting the two, is homologous between the auditory and extrastriate cortices of mammals and the DVR of sauropsids [Karten, 2013].

Subsequent developmental studies confirmed a ventral pallial origin of the DVR, but in mammals they failed to establish the massive tangential migration from the ventral pallium to the dorsal pallium proposed by Karten, undermining the hypothesis of regional homology between the DVR and the mammalian isocortex [Fernández et al., 1998; Puelles et al., 1999; Medina et al., 2011; García-Moreno et al., 2018]. Instead, most of mammalian isocortex was shown to derive from the embryonic dorsal pallium, while the mammalian ventral pallium generates the claustroamygdalar complex. These findings supported previous proposals of pallial evolution based on developmental rather than adult neuroanatomical criteria [Holmgren, 1922; Aboitiz, 1992, 1995; Striedter, 1997, 2005]. Furthermore, we have hypothesized that the expansion of the ventral pallial territory in sauropsids was partly driven by upregulation of the transcription factor Pax6, which facilitates the proliferation of neuronal progenitors during embryogenesis [see also Yamashita et al., 2018]. In addition, mammals underwent overexpression of morphogens secreted by the embryonic cortical hem located in the medial pallium, which determines medial and dorsal pallial development. This process was associated with an increased production of reelin-secreting Cajal-Retzius cells, which regulate radial migration of neurons. Together, cortical hem expansion and Cajal-Retzius cell overproduction helped to restrict the expansion of the ventral pallium, facilitated dorsal pallial growth, and promoted a columnar-laminar organization of the isocortex, as opposed to the globular organization of the sauropsid DVR [Aboitiz, 1999; Aboitiz and Montiel, 2012; Aboitiz and Zamorano, 2013; Montiel and Aboitiz, 2015; see also Bar et al., 2000; Nomura et al., 2008].

\section{Gene Expression and Neural Circuit Similarities among Mammals and Birds}

However, the other aspect of Karten's proposal, that there is homology at the level of neuronal microcircuits, still has widespread support [Wang et al., 2010; DugasFord et al., 2012]. These studies have shown similar input-output microcircuits in the pallium of sauropsids and mammals, and they established similar molecular identities of input-receiving and output neurons in the sauropsid DVR and in the mammalian isocortex. A more recent study [Briscoe et al., 2018] proposes that the so-called intratelencephalic projection neurons, which interconnect different regions of the cerebral hemispheres, also share a gene expression phenotype among mammals, birds, and crocodilians, thus reportedly supporting the notion of an ancestral processing microcircuit that exists in all amniotes. The authors performed a transcription factor expression screen in the embryonic avian mesopallium and nidopallium, and assessed this expression pattern in the brains of alligators and mice. Very interestingly, they found 6 genes that are expressed in the avian mesopallium (ID2, SATB2, NHLH2, FOXP1, BCL11A, and EMX1), which was subdivided into a dorsal component associated with the Wulst (hyperpallium) and a ventral component that is adjacent to the nidopallium. This expression pattern was also detected in the DVR and dorsal cortex of embryonic alligators (stage 25), and in superficial and deep layers of the postnatal mouse isocortex. These mesopallial transcripts were expressed in excitatory neurons and preserve their high expression levels to the adult stage (Fig. 1). However, expression of these factors outside of the studied regions is highly variable, at least in the adult stage (Fig. 1a). Furthermore, although the authors propose that these factors constitute a mesopallial transcription factor network at the protein level, they do not appear to constitute a connected network (Fig. 1b). Available functional association and protein-protein interaction databases allow us to analyze networks of proteins that jointly contribute to a shared function, but this does not necessarily mean they are physically binding each other [Szklarczyk et al., 2017]. For example, in addition to protein-protein interactions, our analysis is able to detect transcriptional activity controlled by cisregulatory elements of noncoding DNA neighboring specific genes. Even allowing for up to 20 proteins/connection directly associated with the input transcriptional factors mentioned above, we find no known interaction between ID2, SATB2 and EMX1 (Fig. 1b).

Intriguingly, the only consistent nidopallium marker gene $(D A C H 2)$ was weakly expressed in the mammalian isocortex and is not present in the basolateral amygdala, suggesting that amplification of this gene's expression is an avian innovation. According to the authors, these findings, taken together, strongly support the hypothesis that a circuit composed of input-receiving, output, and intratelencephalic neurons existed in the last common ancestor of all amniotes. Furthermore, they reportedly support the notion that neuronal cell types can be spatially rearranged during the course of evolution such that they form morphologically very different structures like the neocortex and the DVR, while conserving their molecular and connectional identities.

\section{A Repeated Circuit in Different Pallial Sectors}

Although these findings clearly show important phenotypic and developmental similarities in brain microcircuitry across amniotes, the interpretation of cellular homologies is, in our opinion, open to further discussion. Firstly, it is noteworthy that the mammalian claustroamygdaloid complex, which is embryologically comparable with the sauropsid DVR, has not been considered in the above-mentioned studies [Wang et al., 2010; Dugas-Ford et al., 2012; Briscoe et al., 2018]. Dugas-Ford et al. [2012] homologized input-receiving neurons of layer IV (Eag2/Kcnh5, Rorb/Nr1f2) and output neurons of layer $\mathrm{V}$ (Er81/Etv1, Fezf2/ Znf312, and others) in the cortex of mammals to neurons expressing similar markers in the sauropsidian DVR. However, these markers are also found in the mammalian pallial amygdala (among other regions), suggesting that these are general markers for input-receiving and output neurons in the amniote pallium [Medina et al., 2013]. Likewise, there is a widespread distribution of the intratelencephalic neuron markers in the mammalian hemispheres, including the ventral pallium (Fig. 1a).

Thus, a basic input-output neuronal circuit appears to be replicated across multiple pallial sectors, including the dorsal and the ventral pallium of different species 
[Bosman and Aboitiz, 2015]. This circuit may serve as a basic processing device, synaptically organized to maintain the appropriate excitation-inhibition balance and generating appropriate output to control behavior in simple pallial networks [Bosman and Aboitiz, 2015]. Another recent paper by Briscoe and Ragsdale [2018] is consistent with this interpretation, because it shows a similar circuit comprising inputreceiving, intratelencephalic projections, and output neurons in the mammalian isocortex, the alligator dorsal cortex, and the avian Wulst. As Briscoe et al. [2018] propose, the number and complexity of these circuits have increased independently in birds and mammals, and to a lesser extent in reptiles, through the evolution of additional intratelencephalic projection neurons, which in turn may have led to increased behavioral complexity [see also Tosches et al., 2018].

As discussed above, similar gene expression networks may be expressed in cells with different developmental origins. This happens in repeated body structures like the forelimbs and hindlimbs of tetrapods, the different rhombomeres of the brainstem, and different pallial regions. Thus, the same genetic processes may determine similar cellular phenotypes in say, avian wings and mammalian hindlimbs. However, since the last common amniote ancestor had 4 limbs, avian wings are homologous to the forelimbs, not to the hindlimbs, of mammals. Likewise, although there may be similar excitatory cellular phenotypes in the sauropsid ventral pallium/DVR and the mammalian dorsal pallium/isocortex, embryological evidence indicates that the last common ancestor of amniotes had both a distinct ventral pallium and a dorsal pallium to which the sauropsid DVR and the mammalian isocortex can be traced, respectively [Striedter, 2005; Medina et al., 2011]. Therefore, it may be appropriate to speak of homologous genes that determine similar cellular phenotypes in different organs of two species. However, the problem arises when using these similar gene expression patterns to propose homology between the body or brain parts that cannot be traced back to the same organ in the last common ancestor.

Furthermore, despite the intriguing similarity between avian and mammalian pallial circuits, whether the ancestral amniote had intratelencephalic projection neurons is still an open question, whose resolution will require an analysis of the most basal living amniote species (for example
Sphenodon) and their sister group, i.e., amphibians. Although we cannot study the ancestors' brains directly, fossil evidence indicates that the earliest mammaliaforms had tubular hemispheres, similar in gross morphology to those of present-day amphibians and lungfishes [Kielan-Jaworowska et al., 2004], which may better reflect the ancestral amniote condition. In our opinion, comparative research should be directed to basal living reptiles and amphibians instead of focusing on highly derived species like birds.

Finally, many controversies about homology arise because the ancestors are usually not available for phylogenetic studies, even if some fossil species can provide important insights. In these conditions, the concept of homology is an inference that depends on how one weights the comparative evidence, some scholars focusing on adult structure and others on embryology. Our perspective is that phylogeny is not a sequence of adult stages but a sequence of ontogenies, as proposed by the late Walter Garstang [Holland, 2011]. Thus, evolution will be ultimately understood only by unveiling the developmental transformations that took place in each lineage to yield the "endless forms most beautiful" [Darwin, 1859] of evolving life.

\section{References}

Aboitiz F (1992): The evolutionary origin of the mammalian cerebral cortex. Biol Res 25:4149.

Aboitiz F (1995): Homology in the evolution of the cerebral hemispheres. The case of reptilian dorsal ventricular ridge and its possible correspondence with mammalian neocortex. J Hirnforsch 36:461-472.

Aboitiz F (1999): Comparative development of the mammalian isocortex and the reptilian dorsal ventricular ridge. Evolutionary considerations. Cereb Cortex 9:783-791.

Aboitiz F, Montiel JF (2012): From tetrapods to primates: conserved developmental mechanisms in diverging ecological adaptations. Prog Brain Res 195:3-24.

Aboitiz F, Zamorano F (2013): Neural progenitors, patterning and ecology in neocortical origins. Front Neuroanat 7:38.

Ariëns Kappers CV, Huber CG, Crosby EC (1936): The Comparative Anatomy of the Nervous System of Vertebrates, Including Man. New York, Hafner.
Bar I, Lambert de Rouvroit C, Goffinet A (2000): The evolution of cortical development. An hypothesis based on the reelin signaling pathway. Trends Neurosci 23:633-638.

Belgard TG, Montiel JF (2013): Things change: how comparative transcriptomics suggest the pallium has evolved at multiple levels of organization. Brain Behav Evol 82:150-152.

Belgard TG, Montiel JF, Wang WZ, GarcíaMoreno F, Margulies EH, Ponting CP, Molnár Z (2013): Adult pallium transcriptomes surprise in not reflecting predicted homologies across diverse chicken and mouse pallial sectors. Proc Natl Acad Sci USA 110:1315013155.

Bosman CA, Aboitiz F (2015): Functional constraints in the evolution of brain circuits. Front Neurosci 9:303.

Briscoe SD, Ragsdale CW (2018): Molecular anatomy of the alligator dorsal telencephalon. J Comp Neurol 526:1613-1646.

Briscoe SD, Albertin CB, Rowell JJ, Ragsdale CW (2018): Neocortical association cell types in the forebrain of birds and alligators. Curr Biol 28:686-696.e6.
Darwin C (1859): On the Origin of Species by Means of Natural Selection, or the Preservation of Favoured Races in the Struggle for Life. London, Murray.

Dugas-Ford J, Rowell JJ, Ragsdale CW (2012): Cell-type homologies and the origins of the neocortex. Proc Natl Acad Sci USA 109: 16974-16979.

Fernandez AS, Pieau C, Repérant J, Boncinelli E, Wassef M (1998): Expression of the Emx-1 and Dlx-1 homeobox genes define three molecularly distinct domains in the telencephalon of mouse, chick, turtle and frog embryos: implications for the evolution of telencephalic subdivisions in amniotes. Development 125:2099-2111.

García-Moreno F, Anderton E, Jankowska M, Begbie J, Encinas JM, Irimia M, Molnár Z (2018): Absence of tangentially migrating glutamatergic neurons in the developing avian brain. Cell Rep 22:96-109.

Holland ND (2011): Walter Garstang: a retrospective. Theory Biosci 130:247-258. 
Holmgren N (1922): Points of view concerning forebrain morphology in lower vertebrates. J Comp Neurol 34:391-459.

Jarvis ED, Yu J, Rivas M, Horita H, Feenders G, Whitney O, Jarvis S, Jarvis E, Kubikova L, Buck AEP, Siang C, Martin S, McElroy M, Hara E, Mouritsen H, Chen CC, Wada K (2013): A global view of the functional molecular organization of the avian cerebrum: mirror images and functional columns. J Comp Neurol 521:3614-3665.

Karten HJ (1968): The ascending auditory pathway in the pigeon (Columba livia). II. Telencephalic projections of the nucleus ovoidalis thalami. Brain Res 11:134-153.

Karten HJ (1969): The organization of the avian telencephalon and some speculations on the phylogeny of the amniote telencephalon. Ann NY Acad Sci 167:164-179.

Karten HJ (1997): Evolutionary developmental biology meets the brain: the origins of mammalian neocortex. Proc Natl Acad Sci USA 94: 2800-2804.

Karten HJ (2013): Neocortical evolution: neuronal circuits arise independently of lamination. Curr Biol 23:R12-R15.

Kielan-Jaworowska Z, Cifelli R, Luo Z-X (2004): Mammals from the Age of Dinosaurs. New York, Columbia University Press.

Medina L, Abellán A, Desfilis E (2013): A neverending search for the evolutionary origin of the neocortex: rethinking the homology concept. Brain Behav Evol 81:150-153.
Medina L, Bupesh M, Abellán A (2011): Contribution of genoarchitecture to understanding forebrain evolution and development, with particular emphasis on the amygdala. Brain Behav Evol 78:216-236.

Montiel JF, Aboitiz F (2015): Pallial patterning and the origin of the isocortex. Front Neurosci 9:377.

Montiel JF, Molnár Z (2013): The impact of gene expression analysis on evolving views of avian brain organization. J Comp Neurol 521:36043613.

Montiel JF, Vasistha NA, Garcia-Moreno F, Molnár Z (2016): From sauropsids to mammals and back: new approaches to comparative cortical development. J Comp Neurol 524: 630-645.

Nomura T, Takahashi M, Hara Y, Osumi N (2008): Patterns of neurogenesis and amplitude of reelin expression are essential for making a mammalian-type cortex. PLoS One 3:e1454.

Pfenning AR, Hara E, Whitney O, Rivas MV, Wang R, Roulhac PL, Howard JT, Wirthlin M, Lovell PV, Ganapathy G, Mouncastle J, Moseley MA, Thompson JW, Soderblom EJ, Iriki A, Kato M, Gilbert MT, Zhang G, Bakken T, Bongaarts A, Bernard A, Lein E, Mello CV, Hartemink AJ, Jarvis ED (2014): Convergent transcriptional specializations in the brains of humans and song-learning birds. Science 346: 1256846.
Puelles L, Kuwana E, Puelles E, Rubenstein JLR (1999): Comparison of the mammalian and avian telencephalon from the perspective of gene expression data. Eur J Morphol 37:139150.

Striedter GF (1997): The telencephalon of tetrapods in evolution. Brain Behav Evol 49:179213

Striedter GF (2005): Principles of Brain Evolution. Sunderland, Sinauer.

Szklarczyk D, Morris JH, Cook H, Kuhn M, Wyder S, Simonovic M, Santos A, Doncheva NT, Roth A, Bork P, Jensen LJ, von Mering C (2017): The STRING database in 2017: quality-controlled protein-protein association networks, made broadly accessible. Nucleic Acids Res 45:D362-D368.

Tosches MA, Yamawaki T M, Naumann RK, Jacobi AA, Tushev G, Laurent G (2018): Evolution of pallium, hippocampus and cortical cell types revealed by single-cell transcriptomics in reptiles. Science, Epub ahead of print.

Wang Y, Brzozowska-Prechtl A, Karten HJ (2010): Laminar and columnar auditory cortex in avian brain. Proc Natl Acad Sci USA 107:12676-12681.

Yamashita W, Takahashi M, Kikkawa T, Gotoh H, Osumi N, Ono K, Nomura TD (2018): Conserved and divergent functions of Pax6 underlie species-specific neurogenic patterns in the developing amniote brain. Development 145:8. 